\title{
Screens Using RNAi and cDNA Expression as Surrogates for Genetics in Mammalian Tissue Culture Cells
}

\author{
J. Pearlberg, S. Degot, W. Endege, J. Park, J. Davies, E. Gelfand, J. Sawyer, \\ A. Conery, J. Doench, W. Li, L. Gonzalez, F.M. Boyce, L. Brizuela, \\ J. LaBaer, D. GrueneberG, and E. Harlow \\ Department of Biological Chemistry and Molecular Pharmacology, Harvard Medical School, \\ Boston, Massachusetts 02115
}

\begin{abstract}
We have developed methods for the automation of transfection-grade DNA preparation, high-throughput retroviral preparation, and highly parallel phenotypic screens to establish approaches that will allow investigators to examine in an unbiased manner the roles of proteins in mammalian cells. These methods have been used to raise or lower the levels of individual kinases in individual micro-well cultures either by cDNA or short hairpin RNA expression and will allow investigators to treat mammalian cells in culture in manners that are analogous to genetic screens in yeast. Our proof-of-principle experiments have been performed in human cells using repositories that represent over $75 \%$ of the protein, nucleotide, carbohydrate, lipid, and amino acid kinases in the human genome. These initial experiments have demonstrated the feasibility of two general types of screens. We have performed phenotypic screens to identify proteins with specific roles in a chosen function and genetic interaction screens to establish epistatic relations between different proteins. The results suggest that any phenotype that can be scored by a robust assay in tissue culture is amenable to these types of screens and that interactions between mammalian proteins can be established. These results point to the near-term goal of establishing comprehensive, unbiased screens that will allow queries on the roles of all human proteins.
\end{abstract}

Genetic screens in model organisms have proven to be one of the most important discovery platforms of the last century. Forward genetics using unbiased mutagenesis screens has identified key components of physiological events in a wide array of biological systems (see, e.g., Hartwell et al. 1970, 1974; Hafen et al. 1987; Simon et al. 1991; Nurse et al. 1998; Moon et al. 2004). In many cases, the genes identified in one model organism have led to the identification of related processes in other species not amenable to mutagenesis screens. This has been particularly true in mammalian cells where genetic approaches have been unavailable or difficult. For example, genetic studies in model organisms have proven to be an especially powerful way to find and decipher the function of oncogenes and tumor suppressor genes important in cancer development (Jacks et al. 1994; Forsburg 2001; Xie and Abbruzzese 2003).

Here we describe our approach to genetic screens in mammalian tissue culture cells. Our efforts are animated by the emergence of RNAi technology as well as novel expression vectors, which make it possible to raise or lower the level of a specific protein within essentially any cell that can grow in culture and to study phenotypic changes (Brizuela et al. 2002; Brummelkamp et al. 2002; Hannon 2002; Carpenter and Sabatini 2004; Hannon and Rossi 2004; Paddison et al. 2004; Pearlberg and LaBaer 2004; Rual et al. 2004; Park et al. 2005; Moffat and Sabatini 2006). These tools, when combined with robotic and bioinformatic support platforms, enable these experiments to be performed in a highly parallel high-throughput format. Although changing the levels of a targeted protein is not equivalent to mutagenizing a host cell, expression screens offer a number of advantages in study- ing biological functions. First, the gene under study is known from the onset of the experiment, so no further work is needed to identify and to clone the gene in which a mutation has occurred. Second, the availability of expression RNAi or cDNA vectors facilitates the examination of similar phenotypic changes in a broad range of cells. Third, having the expression sequences in hand provides the tools for immediate biochemical studies, the setting in which most protein function is revealed. Although RNAi and cDNA expression studies do not replace genetic screens, they represent an important avenue for functional studies in mammalian cells.

The ultimate objective for construction of large repositories of sequence-verified cDNAs for open reading frames (ORFs) and RNAi expression vectors will be to cover the entire human proteome. Screens using these large repositories promise to approach the goal of a comprehensive interrogation of the biochemical activities that underlie all cell processes. In the work described here, we have focused on a smaller subset of the proteome, the human kinases. The kinase protein family was chosen for several reasons. First, the kinases are known to play important roles in many or most of the cell decision processes (Hunter 2000). Second, they are frequently mutated in human cancer (Davies et al. 2002; Bardelli et al. 2003; Lynch et al. 2004; Paez et al. 2004). Third, kinases are a common target for drug development, and it is clear that specific small-molecule inhibitors of key kinases can revert characteristics of the tumor cell (Carter et al. 1992; Cobleigh et al. 1999; Druker et al. 2001; Cunningham et al. 2004; see Workman, this volume). Fourth, many laboratories, including our own, have extensive experience in the study of kinase activities and so will be able to follow 
up leads from many screens in an expeditious manner. Finally, although kinase activities have been studied heavily, our knowledge of them is still incomplete. For the work described here, we have been studying protein, nucleotide, carbohydrate, lipid, and amino acid kinases.

In the proof-of-principle studies discussed below, we have learned how to make and use RNAi and cDNA vectors for parallel screens of human cells. This work includes everything from the establishment of robotic procedures for DNA preparation to high-throughput retrovirus production to robust assay development. We show that biological phenotypes of many types can be screened for changes and that shRNA and cDNA expression can be used in combination to look for genetic interactions and to establish epistatic relationships. Importantly, we also have devoted considerable effort to establish methods that allow screens to be performed in a cost-effective manner which will allow these types of assays to be done by many laboratories. Our findings argue that high-throughput functional screens will be an important tool for studying biological activities and promise that comprehensive screens of the entire proteome can be achieved in the near future.

\section{MATERIALS AND METHODS}

Construction of retroviral vectors. pJP1520 was constructed in two steps: The gene encoding the puromycin- $N$ acetyl transferase (PAC) gene was amplified in a PCR and cloned downstream of the $5^{\prime}$ long terminal repeat (LTR) as an NcoI and NotI fragment, creating pJP1519. Next, a fragment was amplified from pLPLNCX (BD Clontech) that comprised the cytomegalovirus (CMV) promoter as well as the loxP recombinational cloning cassette and was then imported into pJP1519 as a NotI and SalI fragment, creating pJP1520. pJP1563, 1564, and 1565 were constructed by removing the PAC gene and replacing it with the blasticidin deaminase (BSDR), hygromycin phosphotransferase (hph), or neomycin phosphotransferase (Neo) genes, respectively. The construction of the epitope-tagged retroviral vectors was performed with a series of PCRs such that overlapping fragments generated fusions of the appropriate epitope tag (either amino- or carboxy-terminal) in the appropriate reading frame.

Recombinational cloning. Purification of Cre recombinase: The coding sequence of Cre recombinase was cloned into the PET28b expression vector (Novagen) so that it contained an amino-terminal 6xHis tag. Next, BL21DE3pLysS cells were transformed with this construct and bacterial lysates were prepared from 1 liter of culture. The mixture was then passed over a $\mathrm{Ni}^{++}$affinity column (Qiagen), the column was washed, and Cre was eluted with imidazole. The eluate was then dialyzed overnight with two changes of medium. From this, approximately $30 \mathrm{ml}$ of active enzyme was generated. All Cre reactions were performed with $200 \mathrm{ng}$ of donor and acceptor vectors, and $1 \mu \mathrm{l}$ of enzyme. After 30 minutes at $37^{\circ} \mathrm{C}$, the reactions were stopped by incubating at $70^{\circ} \mathrm{C}$ for 10 minutes. $5 \mu \mathrm{l}$ was then used to transform Escherichia coli. Competent cells were prepared according to the method of Inoue et al. (1990). After transformation, cells were plated on LB agar plates containing $100 \mu \mathrm{g} / \mathrm{ml}$ ampicillin, $34 \mu \mathrm{g} / \mathrm{ml}$ chloramphenicol, and 7\% sucrose. Colonies were picked the following day and grown in Terrific Broth (TB) containing the same selection cocktail. Plasmid DNA was prepared, and an XbaI diagnostic digestion was performed.

Preparation of plasmid DNA. $1.2 \mathrm{ml}$ of TB with antibiotics was inoculated in 96-well culture blocks and grown overnight in a Multitron shaker. The cells were pelleted, and a standard alkaline lysis was performed. This lysate, in a volume of $850 \mu \mathrm{l}$, was cleared by passage via centrifugation through a $96-$ well $25-\mu$ pore size filter plate (Whatman). The filtrate was then loaded onto the anion exchange (AIEX) resin plate. The AIEX plates are prepared in advance by pouring (either as slurry or powder) resin into a $25-\mu$ filter plate. The mixture is spun at low speed for 3 minutes. The resin is then washed, and a high-salt elution step is performed. The DNA is then precipitated by the addition of an equal volume of isopropanol and the DNA is pelleted by centrifugation, washed with $70 \%$ ethanol, and dried. The pellet is resuspended in $100 \mu \mathrm{l}$ of TE $(10 \mathrm{mM}$ Tris, $\mathrm{pH} 8$; 1 mM EDTA), and DNA is quantitated using the Hoechest Dye H33258. The DNA concentration was normalized to $5 \mu \mathrm{g} / \mu \mathrm{l}$ and stored at $-20^{\circ} \mathrm{C}$.

Generation of virus. Generation of Moloney murine leukemia virus (MMLV): 293 T cells were seeded at a density of 65,000 cells per well in a volume of $100 \mu \mathrm{l}$ in 96well plates. The following day, cells in each well were transfected with100 ng of MMLV retroviral vector, $75 \mathrm{ng}$ of a gag-pol expression construct, and $50 \mathrm{ng}$ of a vesicular stomatitis virus G (VSVG) expression construct using Fugene 6 transfection reagent according to the manufacturer's suggestions (Roche). The plates were immediately centrifuged for 30 minutes at $2000 \mathrm{rpm}$ and then placed in a $37^{\circ} \mathrm{C}$ tissue culture incubator. The following morning, medium was removed and $200 \mu$ of fresh medium was added. The plates were then placed in a $32^{\circ} \mathrm{C}$ tissue culture incubator, and medium was harvested, stored at $4^{\circ} \mathrm{C}$, and replenished daily for 1 week. At the end of the collection period, the viral supernatants for each daily collection were aliquoted in 100- $\mu$ l volumes and stored in 96-well plates at $-20^{\circ} \mathrm{C}$.

Generation of lentivirus: $293 \mathrm{~T}$ cells were seeded in 96well plates at a density of 55,000 cells per well in a volume of $100 \mu \mathrm{l}$. The following day, cells in each well were transfected with $100 \mathrm{ng}$ of lentiviral vector, $100 \mathrm{ng}$ of a gag-pol-rev expression plasmid, and $20 \mathrm{ng}$ of a VSVG expression plasmid, using Fugene 6 transfection reagent according to the manufacturer's suggestions (Roche). The plates were immediately centrifuged for 30 minutes at $2000 \mathrm{rpm}$ and then placed in a $37^{\circ} \mathrm{C}$ tissue culture incubator. Viral supernatant was collected $(200 \mu 1)$ twice daily for 3 days starting the day following transfection. At the end of the collection period, the virus was aliquoted and stored at $-20^{\circ} \mathrm{C}$.

Viral infections: All viral infections were performed the day after plating cells. From $3 \mu \mathrm{l}$ to $10 \mu \mathrm{l}$ of thawed viral supernatant (either MMLV or lentivirus) was added to 
each well in the presence of $8 \mu \mathrm{g} / \mathrm{ml}$ polybrene. The plates were spun for 30 minutes at $2250 \mathrm{rpm}$ and then placed in a $37^{\circ} \mathrm{C}$ tissue culture incubator. The following day, the medium was removed and fresh medium was added.

Growth and proliferation assays. For the cell outgrowth assay in SKBR3 cells, cells were infected with either lentivirus or MMLV and 24 hours postinfection were treated with $1 \mu \mathrm{M}$ Iressa (provided by Astra Zeneca). After 2 weeks, the wells were visually inspected, and those wells in which the cells displayed obvious growth were scored as positive. For quantitative proliferation assays, after approximately 4 days of either drug treatment or infection, a one-tenth volume of a Resazurin stock solution (stock $150 \mathrm{mg} /$ liter, prepared in PBS) was added to the well. After 4-12 hours, a fluorometric reading was performed in a TECAN plate reader set to an excitation wavelength of $535 \mathrm{~nm}$ and an emission wavelength of $590 \mathrm{~nm}$.

Scoring of kinases. Essential kinases: Each duplicate data point was taken in average and then measured against the average value for the entire experiment. Those data points whose value was less than $50 \%$ of the average for the experiment were designated as essential kinases.

Drug-sensitizing kinases: In this case, to score as a hit, the effect of the shRNA itself, in the absence of drug, was taken into account. In the absence of drug, the Resazurin value for a given well was required to be at least $75 \%$ of the average for the entire no-drug-treatment arm of the experiment. Those shRNAs that passed this test were then analyzed in the context of the drug-treatment arm of the experiment. To score as a hit, the Resazurin value was required to be at most $50 \%$ of the average for the entire drug-treatment arm of the experiment.

\section{RESULTS}

To begin the development of high-throughput functional screens, we have made several fundamental decisions about acceptable technical approaches. Two goals are particularly important to our strategy. First, we have chosen to use viral vectors to ensure easy and efficient transduction of expression constructs. Transfection of siRNAs themselves, shRNA vectors, or cDNA vectors is possible, but this greatly limits the types of cells that can be studied, and adjusting expression levels is extremely difficult. In addition, expression vectors of all types are easily shared and, consequently, compared across labs and experimental systems. Second, we have devised methods that allow each construct to be tested independently. Pooling strategies are powerful in many settings, but they are not suitable for screens in which the phenotypic readout comprises some aspect of a growth defect, such as cell cycle arrest. An additional limitation relates to working with pooled libraries. Since the starting library is a mixture of species, the precise identity of a clone that scores positive in a screen is anonymous: It must then be determined by retrieving that clone from the cell and sequencing the insert. Moreover, it is possible to extract additional information from a genetic screen from those clones that fail to elicit the phenotype in question only when the identity of all clones is known prior to executing the experiment.

There have been two major stages to developing highthroughput functional screens in our lab. The first stage centered on the development of methods and techniques to produce large numbers of expression clones with sufficient ease and of adequate quality to use in parallel screening assays. Another goal of these technical approaches has been to produce these reagents at low enough cost to make screening assays possible for most academic research labs. The second stage has been the development of tissue culture methods and screening assays to utilize these reagents. Both of these stages have been completed successfully; a brief outline of our strategy and results is presented below.

\section{Stage 1: Expression Clones}

The basis of this system rests on the ability to effectively transfer a gene (or knockdown shRNA) to a variety of cell types. This process must be efficient, reliable, generalizable, selectable, and, preferably, trackable. To this end, a retroviral vector with the appropriate markers fulfills these requirements and makes this an ideal method for high-throughput gene delivery. An additional feature that makes this strategy for gene transfer attractive is that the manipulations required to produce retroviral stocks do not involve multiple complicated steps, making the adaptation to a high-throughput scale of operation possible.

The viral vector employed for our shRNA experiments is a lentiviral design developed by a collaborative group of investigators, collectively known as the RNAi Consortium (TRC) (http://www.broad.mit.edu/genome_bio/trc/). The TRC generously provided the shRNA constructs used here prior to publication. In this system, the shRNA is expressed from the human U6 promoter, downstream from which resides an expression cassette for the PAC gene between the LTR elements, so that transduced cells can be selected for upon the addition of puromycin. The expression cassette is designed so that the processed duplex RNA is 21 nucleotides in length. As is the case with other shRNA libraries, this clone collection is degenerate, with approximately 4-5 shRNAs, each targeted to a specific gene (Moffat et al. 2006). The shRNA set in our possession designed to target roughly 450 kinases numbers some 2500 discrete clones.

In our laboratory, we have generated a Moloney retroviral cDNA expression vector with recombinational cloning elements and have validated its stability during cloning manipulations. We have also performed functional validation studies in mammalian cells to ensure that the retroviral and expression elements work as intended. We went to some length to characterize this vector prior to implementing it into our cloning and experimental pipelines because it would be used for the expression of thousands of cDNAs in the course of our work, as we describe in some detail here. This vector was designed to accommodate the Creator recombinational cloning system, based on cre recombinase, developed by BD Clontech (http://www.clontech. com/clontech/index.shtml). This design took advantage of the cDNA repository developed by the Harvard Institute of 
Proteomics (HIP www.hip.harvard.edu). HIP's founding principle was to create and distribute a catalogued collection of full-length, sequence-verified cDNA clones, available for use in a wide variety of settings.

As of this writing, HIP has generated approximately 5500 full human cDNAs, all of which have been sequence-verified. These gene ORFs have been cloned into a master recombinational cloning vector, which facilitates transfer into a variety of expression vectors. From this collection, we have focused our interest on the complement of human kinases. From among the many Moloney retroviral vectors we tested, we found that the vector pMMPf2 (provided by Richard Mulligan of The Harvard Gene Therapy Initiative, Harvard Medical School, http://hgti.med.harvard.edu) proved to be the most stable and least prone to rearrangements in E. coli during cloning manipulations. Therefore, we decided to incorporate the features necessary for our experiments into this vector. pMMPf2 is a Type $\mathrm{C}$ gammaretrovirus vector whose LTRs comprise elements of both MMLV and murine proliferative sarcoma virus. As with other retroviral vectors used for gene delivery, the gag, pol, and env genes have been removed. We have modified this vector in the following ways: PAC, which confers resistance to puromycin, was inserted as an NcoI and NotI fragment downstream of the 5'LTR. Growing cells in the presence of puromycin will allow selection of those cells that have been successfully infected. In a second step, a loxP recombinational expression cassette, compatible with the Creator recombinational cloning system as used at HIP, was amplified in a PCR and introduced as a NotI and XhoI fragment between the LTRs, just downstream from the PAC gene, generating the plasmid, pJP1520 (Fig. 1A). We have tested pJP1520 to ensure that it is stable during procedures performed in E. coli and that it remains stable during subsequent propagation. For reasons that remain obscure, pJP1520 has performed remarkably better than other vector constructs in tests for arrangements of the various selection and expression elements that we required. These tests included the isolation of multiple independent isolates with transferred expression ORFs and long-term culture. Other vectors showed significantly increased levels of recombination in E. coli. Of note, pJP1520 showed almost no recombinations, even when these procedures were carried out in the recA-positive bacterial strain MM294.

To complement pJP1520, three additional Moloney retroviral vectors, each with a different selectable marker (that for hygromycin, blasticidin, or G418 [neomycin]), have been constructed. An additional series of vectors, also based on pJP1520, was designed so that either an amino- or carboxy-terminal epitope tag (either flag, myc, $\mathrm{HA}$, or taptag) is appended to the ORF after recombinational transfer (Fig. 1B). We anticipate that these vectors will prove useful in a variety of settings.

To date, we have transferred over 500 full-length kinase cDNAs generated by HIP into pJP1520 as well as its blasticidin-markered sibling, pJP1563. The initial cloning of these kinases, as well as their recombinational transfer, was performed in 96-well format. For these cloning operations, we use Cre recombinase that we have made in our laboratory; it performs as well as any commercial source we have tested and is substantially cheaper. In addition, we do not use commercially prepared competent cells. We

A

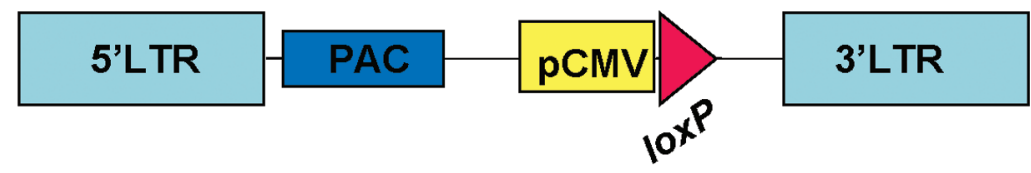

B

\begin{tabular}{|c|c|c|}
\hline Name & Resistance Marker & Epitope Tag (position) \\
\hline pJP1520 & puromycin & none \\
\hline pJP1563 & blasticidin & none \\
\hline pJP1564 & Hygromycin & none \\
\hline pJP1565 & Neomycin & none \\
\hline pJP1536 & puromycin & 3xFlag (N-ter) \\
\hline pJP1537 & puromycin & Myc (N-ter) \\
\hline pJP1538 & puromycin & HA (N-ter) \\
\hline pJP1539 & puromycin & TAP-tag (N-ter) \\
\hline pJP1540 & puromycin & 3xFlag (C-ter) \\
\hline pJP1541 & puromycin & Myc (C-ter) \\
\hline pJP1542 & puromycin & HA (C-ter) \\
\hline pJP1543 & puromycin & TAP-tag (C-ter) \\
\hline
\end{tabular}

Figure 1. Retroviral expression vectors with recombinational cloning elements. $(A)$ Schematic drawing of the relevant elements of the pJP1520 vector. Included in the diagram are the LTR elements, a 600-bp fragment of the human CMV immediate-early enhancer/promoter, the PAC gene, and the 34-bp loxP element. (B) Retroviral vectors derived from pJP1520. 
have found that competent cells prepared in-house are as efficient as any commercial source and provide a remarkable cost savings. Each kinase cDNA ORF is sequenceverified after it is cloned into the master recombinational cloning vector. All cloning steps are tracked with a laboratory information management system (LIMS). After the transfer reaction into either of the two Moloney retroviral vectors, a restriction digestion is performed to assess the success of the reaction. We have found that on first pass, we achieve a success transfer rate of approximately $90 \%$. All of our clones are stored as glycerol stocks in a Biobank robotic freezer storage system.

\section{Stage 1: High-Throughput Generation of Transfection-grade DNA}

Perhaps the single most challenging step we have faced is the conversion of a glycerol stock into highly transfectable plasmid DNA. Although a number of highthroughput plasmid DNA preparation kits are available from commercial vendors, we have found them to be expensive, and they deliver DNA that shows low rates of transfection compared to other sources. Therefore, we have developed our own methods, based on standard alkaline lysis, to prepare plasmid DNA of sufficient purity for transfection of mammalian cells (Fig. 2A). A few key points of the DNA preparation methods are highlighted here, and the details of these methods and protocols will be published elsewhere. Typically, we grow cultures in a 96-well culture block in a volume of $1-1.5 \mathrm{ml}$ of TB per well. Then a standard alkaline lysis step is performed, and the lysates are cleared by passage through a filter plate. In our experience, either centrifugation or a vacuum can be applied at this step. We then tested a number of anion exchange resins and found two that worked well: the weak DEAE anion exchanger, Nucleobond, and the strong QAE anion exchanger, cholestyramine. Nucleobond, developed by Machery Nagel, is the resin used by Qiagen in their Maxiprep kits. The cholestyramine resin binds plasmid DNA exceptionally well under our conditions, and we have found it to be an inexpensive reagent that works well. We load either of the resins into the wells of a 96-well filter plate and perform the wash and elution steps either by vacuum or by centrifugation. We have tested a variety of methods to then remove the salt from the eluate. We currently use alcohol precipitation as the method of choice. We have found that the transfection efficiency of the plasmid DNA prepared by our high-throughput miniprep methods is equal to that of plasmid DNA prepared with a Qiagen Maxiprep kit (Fig. 2B). From a 1-ml culture, our yields are typically in excess of $10 \mu \mathrm{g}$. This provides enough DNA for 200 transfections. Without robotic assistance, 12 plates - corresponding to 1200 minipreps - can be processed in a day by one person. With robotics, this scale can be increased five- to tenfold.
A
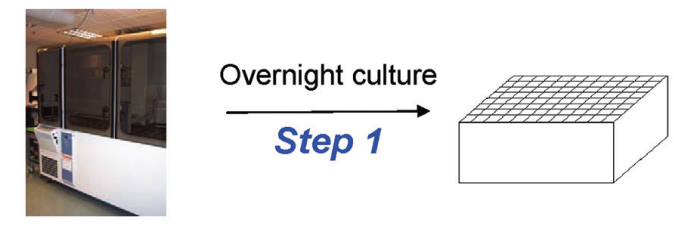

Glycerol stocks

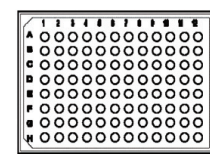

96 well DNA
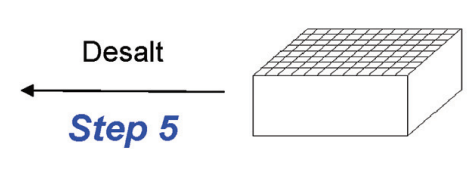
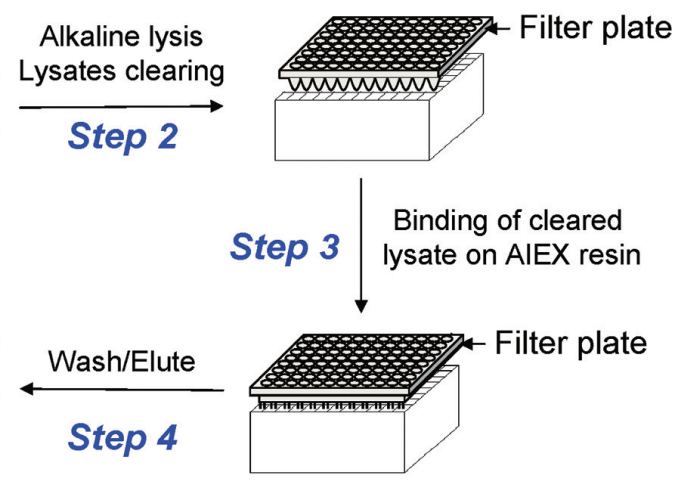

B

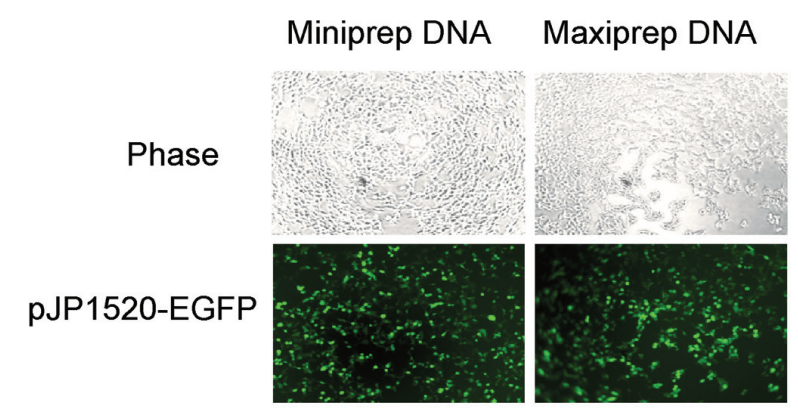

Figure 2. Work flow for high-throughput minipreps. (A) Simplified scheme of DNA minipreps. All of the shRNA and cDNA expression clones glycerol stock are stored in Thermo Electron Biobank freezer system. From these glycerol stocks, bacteria are grown overnight in $1.2 \mathrm{ml}$ of TB medium (Step 1). After pelleting, an alkaline lysis step is performed and the supernatant is cleared by centrifugation through a filter plate (Step 2). The cleared lysates are then loaded on a second filter plate containing an AIEX resin (Step 3). After a wash step, DNA is eluted (Step 4). The transfection-quality miniprep DNA is then obtained by a final desalting step (Step 5). (B) Comparison of inhouse miniprep transfection efficiency to Qiagen Maxiprep DNA. $100 \mathrm{ng}$ of pJP1520 DNA obtained using either our miniprep protocol or Qiagen Maxiprep was transfected in 293 T cells. 48 hours later, the transfection efficiency was assessed by GFP expression. 


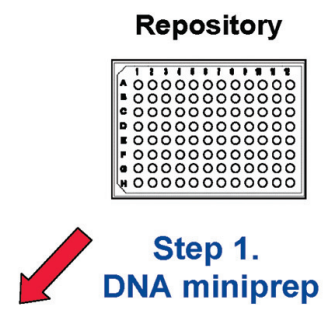

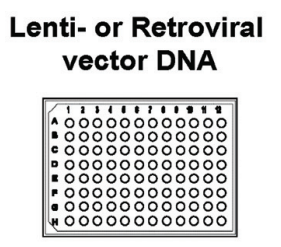

Step 2. Transfection

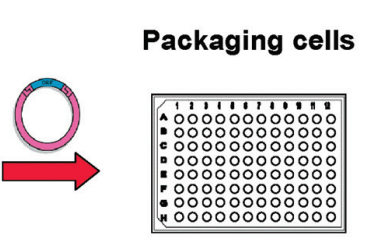

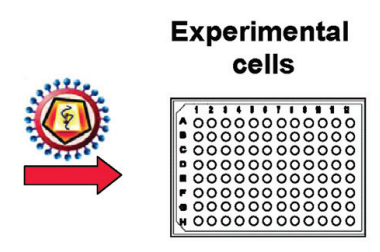

Step 3. Transduction

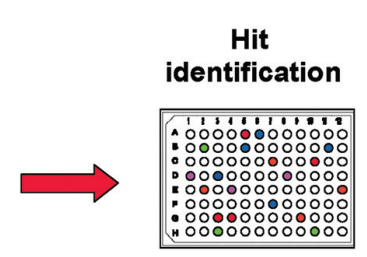

Step 4. Assay

Figure 3. Essential steps to perform screens in mammalian cells. The work flow to conduct screens into mammalian cells starts with the conversion of bacterial glycerol stocks from the repository into DNA using our 96-well miniprep DNA protocol. After normalization of DNA concentration, 293T cells are co-transfected with the DNAs of interest, the packaging constructs (Gag/pol and $\mathrm{Gag} / \mathrm{pol} / \mathrm{rev}$ for the MMLV retrovirus and lentivirus, respectively), and VSVG protein to produce pseudotyped viruses. These viruses are then used to transduce the experimental cell line in 96-well or 384-well format.

\section{Stage 1: High-Throughput Generation of Retrovirus Stocks}

From the high-quality plasmid DNA we have in hand, we are able to generate high-titer virus. The work flow is shown in Figure 3. Transfections are performed in 96-well format, and viral supernatant is collected, aliquoted, and frozen. The transfection and all downstream liquid-handling steps are performed robotically with a Sciclone ALH3000 robot (Caliper Life Sciences). Procedurally, the generation of lentivirus and MMLV retrovirus is very similar in our laboratory, and we have spent some effort to optimize each system. Starting from $100 \mathrm{ng}$ of viral vector DNA, we typically generate roughly $0.8 \mathrm{ml}$ of lentivirus and $1.5 \mathrm{ml}$ of MMLV retrovirus. Using test viruses that express the enhanced green fluorescent protein (EGFP), we have determined by flow cytometry that the titer of the lentivirus is slightly below $10^{7}$ per ml. For the MMLV retrovirus, it is approximately $10^{6}$ per $\mathrm{ml}$. Since our screens are performed for the most part in 384-well plates, with approximately $400-800$ cells per well, we find that we can use 3-5 $\mu$ l of virus per well. For both the lentiviral and MMLV platforms, we pseudotype our virus with the VSVG protein, which imparts a broad degree of flexibility to our experimental system. As a biosafety precaution, our Sciclone robot is surrounded by a custom laminar flow hood (Flow Sciences), with an airflow system that generates negative pressure. With these methods, virus production is robust, and the limiting factor in the production of screening reagents is the DNA preparation.

As we have developed and refined the methods described above to generate virus, we have also in parallel begun to perform some pilot genetic screens. Here we present some examples of the types of screens we have developed. Because the data sets generated by these approaches are large, the detailed findings of these screens will be presented elsewhere.

\section{Stage 2: Identification of Essential Kinases in Lung Cancer Cell Lines}

To begin to develop a framework to understand key signaling molecules and their attendant pathways in lung cancer tumor cell lines, we initiated a series of screens designed to identify the set of essential kinases in a number of non-small-cell lung cancer cell lines. The results of these experiments from four such cell lines (A549, NCIH23, NCI-H358, NCI-1299) are discussed here. These efforts utilized the lentiviral shRNA library described above. As mentioned previously, approximately 450 kinases were interrogated, and with a library degeneracy of 4 or 5 shRNA hairpins targeted to each kinase. This corresponds to roughly 2500 discrete lentiviral shRNA clones from which plasmid DNA was prepared and virus was generated. For these experiments, lentiviruses were prepared in 96-well format and were then used to transduce the lung tumor cell lines in 384-well format. Each infection was performed in quadruplicate, and then half of the wells were treated with puromycin selection and half were not. The cells were then grown for 4 days, and a proliferation assay was performed. The puromycin selection allowed us to define in a general sense the efficiency of viral transduction. In the scatter plot shown in Figure 4, those wells treated with puromycin were plotted against their untreated counterparts, and the results indicate that the viral infections worked well. From the set of wells not treated with puromycin we were able to determine those shRNA molecules that inhibited proliferation, i.e., identify essential kinases. As shown in Table 1, roughly 50-100 essential kinases were identified from each of four cell lines tested. In total, from among the four cell lines tested, 177 essential kinases were found. Roughly $60 \%$ of those essential kinases were not shared across the cell lines tested. A small number of kinases (8) was found to be essential in all four cell lines (Fig. 5). In 10\% of the cases when an 


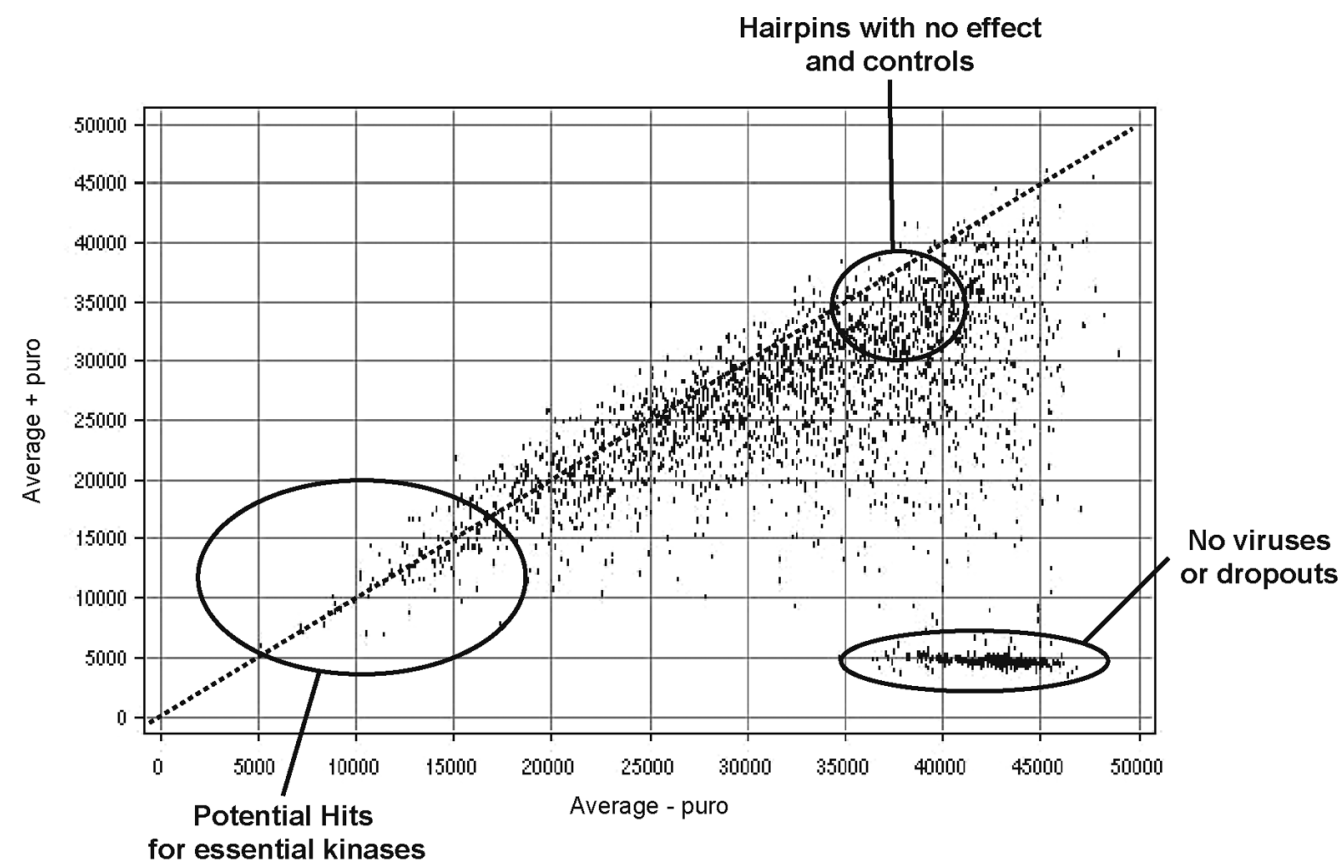

Figure 4. Scatter plot of data points from essential kinase screen in NCI-H23 lung cancer cell line. In each experiment, infection was performed in quadruplicate. From among the quadruplicate wells, two wells received puromycin and two did not, and after several days, cell proliferation was assayed. The duplicate points for each condition were averaged and plotted against each other. The $x$ and $y$ axes represent the raw Resazurin values from the fluorometric readings. This scatter plot shows the correlation between the two conditions (presence or absence of puromycin). An $\mathrm{r}$ of 1 is indicated by the dashed black line. Hits are identified as indicated in the figure and described in Materials and Methods.

shRNA scored as a hit, additional hairpins targeted to different regions of that gene also scored positive. It is our expectation that as more cell lines are tested in this manner, we may be able to determine those kinases whose expression is required by a broad spectrum (or perhaps all) of tumor lines. In addition, we may be able to discern patterns based on tissue histology or the presence of particular genetic alterations (e.g., mutations in EGFR or Kras).

\section{Stage 2: Genetic Modifier Screens}

A second type of assay, also based on the same phenotypic readout, is a genetic modifier screen designed to identify those kinases whose expression levels modify the response of tumor cell lines to various chemotherapeutics. These pilot efforts have been initiated in a set of breast cancer and lung cancer cell lines, and various subsets of our shRNA set as well as our cDNA set have been used for these experiments. Our initial studies began with the breast cancer cell line SKBR3. This cell line displays moderate sensitivity to the EGFR inhibitor Iressa, with an $\mathrm{IC}_{50}$ of ap- proximately $1 \mu \mathrm{M}$ (data not shown). In this case, a small screen in which approximately 250 kinases, prepared as retrovirus and overexpressing individual kinases as described above, were examined to determine which conferred increased resistance to Iressa. Here we employed a simple yet robust assay in which we determined those wells in which there was obvious cell growth after a period of 2 weeks (Fig. 6). Of the kinases tested, about 12 enabled this cell line to grow in the presence of $1 \mu \mathrm{M}$ - and in some cases $10 \mu \mathrm{M}$ - Iressa for several weeks. It is worth noting that a number of those kinases that scored positive in this assay bear an obvious relationship to the action of EGFR and the EGFR pathway. For example, Her2, ErbB3, and Akt all render SKBR3 cells resistant to Iressa (shown as Wells B10, C3, and E9, respectively, in Fig. 6). In addition, we have identified a number of kinases with no obvious relationship to EGFR or the EGFR pathway that increase the resistance of SKBR3 cells to Iressa.

In a similar vein, we also identified particular kinases that when underexpressed, via shRNA-mediated knockdown, render SKBR3 cells resistant to Iressa. In this pilot

Table 1. Overview of Essential Kinase Screens in Four Different Lung Cancer Cell Lines

\begin{tabular}{lcccc}
\hline Cell line & A549 & NCI-H23 & NCI-H358 & NCI-H1299 \\
\hline Histological type & adenocarcinoma & non-small-cell & adenocarcinoma & large-cell \\
& & carcinoma & 81 & carcinoma \\
Total number hairpins scoring & 115 & 3 & 1 & 50 \\
$\quad$ triplicate hairpins for the same gene & 5 & 7 & 8 & 0 \\
$\quad \begin{array}{l}\text { duplicate hairpins for the same gene } \\
\text { Total number of different genes }\end{array}$ & 9 & 69 & 70 & 4 \\
\hline
\end{tabular}




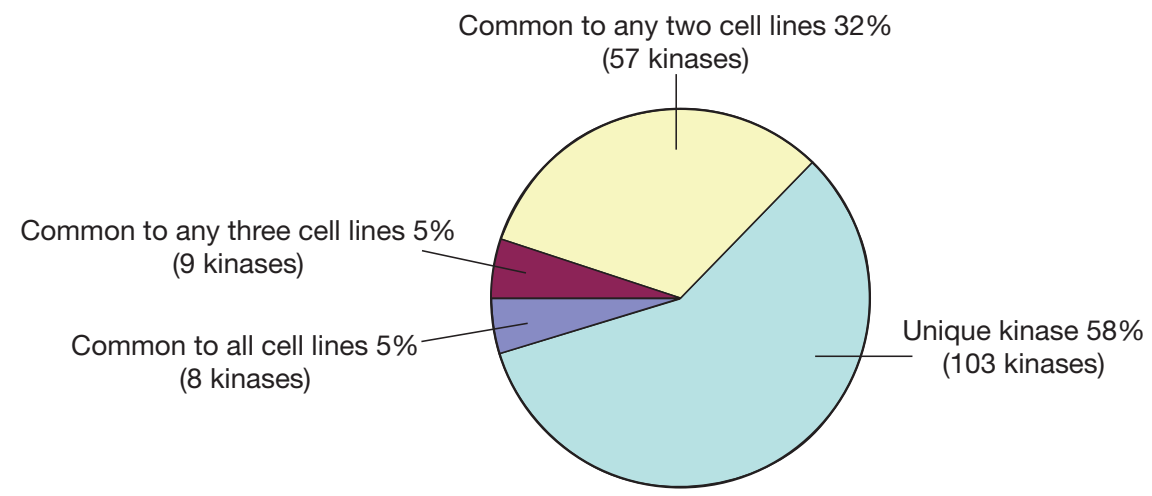

Total number of kinases: 177

Figure 5. Essential kinases shared among four cell lines.

experiment, a few hundred shRNA clones, corresponding to roughly 100 genes, were assayed and scored as described above. In this scenario, the shRNA cassette was introduced into SKBR3 cells via lentiviral infection. As was the case for the cDNA expression experiments described above, after infection, the cells were grown in the presence of $1 \mu \mathrm{M}$ Iressa for 2 weeks. Roughly 10 kinases, when targeted for knockdown, allow SKBR3 cells to grow in these conditions (for examples, see Fig. 6). In a few cases, two shRNA clones, each corresponding to a different region of the same gene, that conferred resistance to Iressa were identified. Since the set of kinases targeted in this small shRNA experiment for the most part did not overlap those kinases tested in our cDNA expression experiment above, we are unable to determine whether in the case where shRNA renders a cell resistant to our test drug, there is a corresponding cDNA that renders the cell sensitive. We anticipate that as our test sets become more complete, we will encounter examples of this. Since it is known that GSK3B, at least in some contexts, antagonizes the action of Akt, it is interesting that GSK3B scores when targeted for knockdown, given that Akt when overexpressed scores similarly (shown as Well A5 in Fig. 6). As of this writing, we have not determined the degree of knockdown elicited by the shRNA clones used in our experiments. These efforts are ongoing, and as we accrue information regarding the efficacy of knockdown by particular shRNA clones in our possession, we shall eliminate from our experiments those clones that are not effective. In addition, this information will impart additional meaning to those clones that do not score despite the fact that they result in mRNA knockdown.

We have also performed a similar series of experiments with our cDNA expression clones in a set of non-

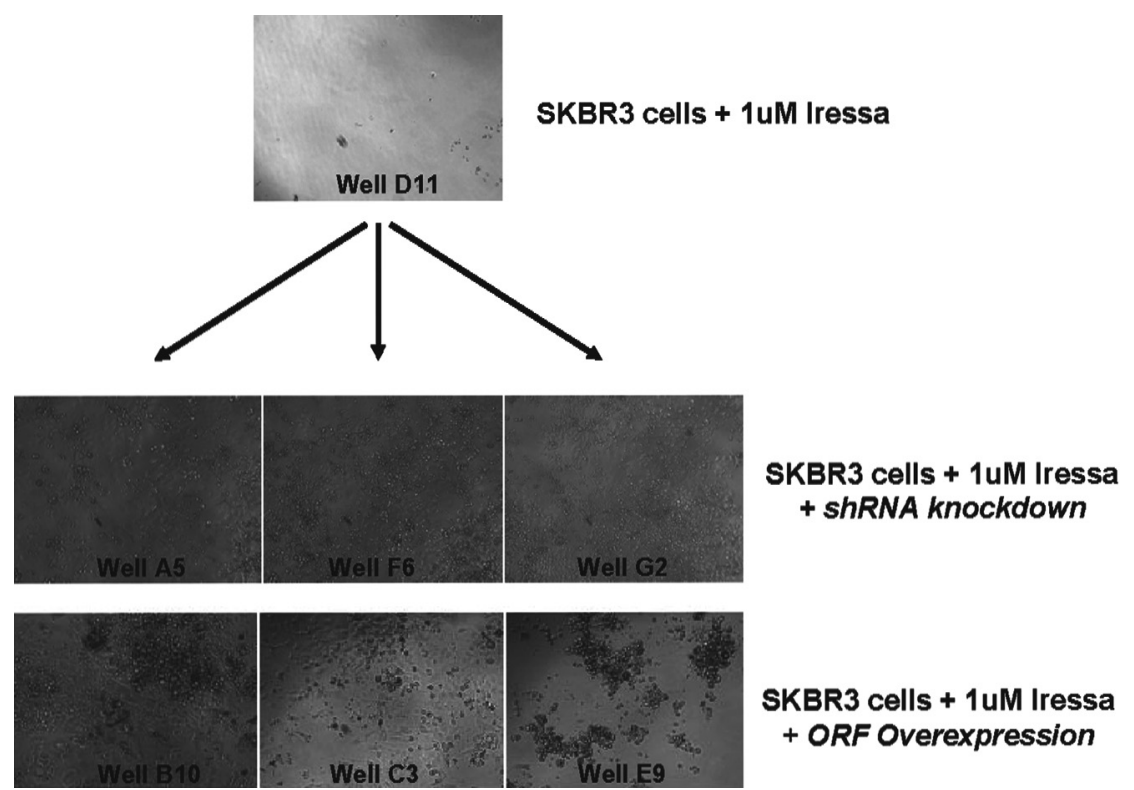

Figure 6. Modulation of Iressa sensitivity. SKBR3 cells were cultured in the presence of $1 \mu \mathrm{M}$ Iressa for 2 weeks (top photograph). Cells rendered resistant to $1 \mu \mathrm{M}$ Iressa by either shRNA knockdown or cDNA overexpression of particular kinases (bottom photographs). 
small-cell lung cancer lines. In some cases, the starting cell line, such as HCC827 or H1650, displayed inherent sensitivity to Iressa. Therefore, these two lines were used to identify kinases that, when overexpressed, increased resistance to Iressa. As was the case with SKBR3 cells, a number of kinases relating to EGFR and its downstream effectors, such as Akt, ErbB3, and HER2, scored in either or both of these cell lines. In addition, a small number of kinases, whose relation to EGFR remains obscure, or at best tenuous, were identified. The fact that in these pilot screens we identify kinases whose properties obviously relate to the action of the drug as well as those do not provide a measure of confidence that our screening system will uncover novel proteins whose function is physiologically relevant to the action of Iressa (or for that matter, any other test drug).

\section{Stage 2: Genetic Suppression}

One of the most powerful experiments that can be undertaken with our approach is that of genetic suppression. Thus, we attempted the following simple experiment. From some of our initial shRNA studies, we identified ErbB3 as an essential kinase in a number of our cell lines. We were then interested in identifying those kinases that when overexpressed would rescue - or suppress - the phenotype elicited by either of two ErbB3 shRNA knockdown cassettes (Fig. 7). This experiment took advantage of the various selectable markers carried by our viral vectors. In this case, HeLa cells were seeded in 96-well plates and the cells were transduced with virus (based on pJP1563), so that the cells in each well were engineered to overexpress an individual kinase (roughly 250 kinases were surveyed). The cells were then put into blasticidin selection. The day after, either of the two ErbB3 shRNA cassettes was introduced into the cells via lentiviral transduction. Puromycin selection was then instituted. Ordinarily, knockdown of ErbB3 would result in death of HeLa cells. From among the 250 kinases that were overexpressed, we identified one kinase, MAP3K14, that overcame the killing mediated by ErbB3 knockdown. This result argues that this line of experimentation will allow us to establish genetic relationships among genes and pathways much in the same way as has been done in yeast and other model organisms for the past several decades.

\section{DISCUSSION}

Experiments with mammalian tissue culture cells have proven to be an excellent system for biochemical studies. Methods to lyse cells, purify components, and reassemble cellular processes in a test tube have led to major advances in our understanding of DNA synthesis, transcription, translation, and many other key aspects of cell physiology. Similarly, affinity methods to rapidly purify individual proteins and examine the isolated proteins for changes in association and modifications have been a staple of building signal transduction pathways and understanding many aspects of regulation. Imaging methods provide ever-higher resolution of the spatial and temporal location of proteins. One approach that has been missing from the tools used to study mammalian cells in culture is any process that resembles genetics. Mutagenesis screens allow researchers to identify key genes in an unbiased manner and can approach comprehensive coverage of the entire genome. The availability of comprehensive li-

A

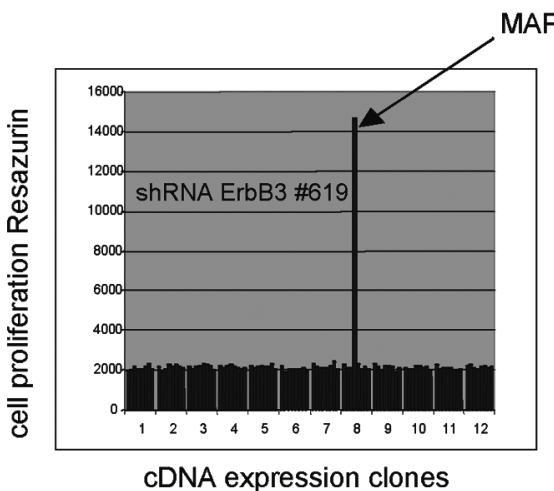

MAP3K14

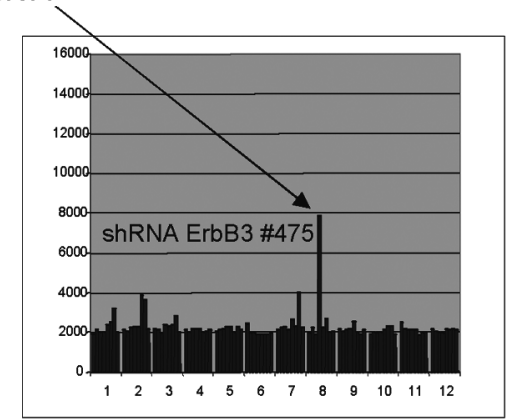

cDNA expression clones

B

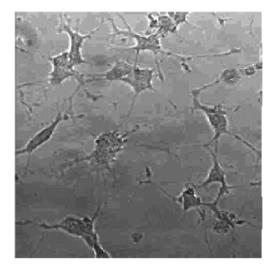

ShRNA ErbB3 \#619

+ MAP3K14

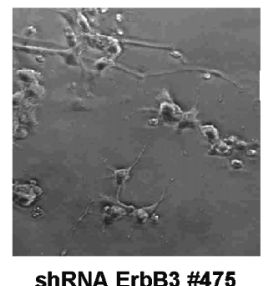

shRNA ErbB3 \#475

+ MAP3K14

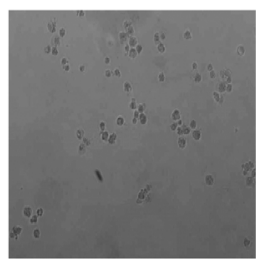

shRNA ErbB3

+ well next door

Figure 7. Example of genetic suppression. $(A)$ Map3K14 rescue of ErbB3 shRNA-mediated killing of HeLa cells. From among 250 kinases tested, Map3K14 was found to suppress the growth defect elicited by either of two ErbB3 shRNA knockdown cassettes (shRNA ErbB3 \#475 or shRNA ErbB3 \#619) in HeLa cells. Cell growth was determined using a Resazurin-based proliferation assay. $(B)$ Cell images were collected by phase-contrast microscopy. 
braries of shRNAs and cDNA expression vectors will allow screens that approach the characteristics of genetic screens. With these tools, the levels of a particular protein can be raised or lowered within a target cell in assay formats that closely resemble genetic mutation screens.

It is worth noting that although the comparison to genetics helps one understand how to use and plan experiments using expression screens, there are true differences between these approaches. Mutagenesis in theory can hit any gene or regulatory sequence. High-throughput functional screens only interrogate the proteins represented in the repository. This collection is increasing but is still short of comprehensive, and in the proof-ofprinciple work described here, we have examined only the kinase protein family. Mutations can change the function of a protein in many fashions, including loss of function and gain of function. High-throughput screens rely on differences caused by increasing or decreasing the levels of a particular protein. Importantly, mutations directly affect only the altered protein, whereas expression of shRNAs or cDNAs can have effects off target. It is now well understood that shRNAs can affect mRNAs that are not the designed target, and high levels of proteins expressed from cDNAs can lead to unexpected protein-protein associations that can act as dominant interfering interactions. Both shRNA and cDNA off-target effects can be eliminated in secondary screens by use of multiple hairpins to the same target in the case of shRNAs, and by mapping the effector regions for cDNAs. The use of high-throughput functional screens has several advantages over mutagenesis. The most important is that from the completion of the screen the exact protein under study is known. Mutagenesis commonly requires the mapping and cloning of the lesion before the gene in question can be identified. All of these facts distinguish the use of expression screens from classic mutagenesis. However, like mutagenesis, the goal of functional screens is to pinpoint a gene or protein that can serve as a starting point for more detailed experimentation. High-throughput functional screens now open this experimental door for investigation of tissue culture cells.

A reasonable goal for the next several years will be to build shRNAs and cDNAs for every protein in human cells. As these reagent-building projects progress, and as these tools get used in screens, extensive analysis of their actions can be assembled and used to refine the reagent set with the goal of having a validated reagent set. With these developments, the genetic information gleaned from the sequencing efforts of the last decade can be converted into powerful reagents that will allow each protein to be tested for its role in chosen cellular processes.

What types of assay formats and strategies can be approached with high-throughput functional screens? We have chosen to approach the development of these reagents with three general goals. First, we have sought to develop methods that will allow each protein to be tested separately. Other investigators have shown the value of using pools of clones. Pooling strategies are cost- and labor-efficient but often limit assay design methods. Testing each clone individually allows more types of screens to be available for assay, promotes validation of each reagent, and leads to the possibility to record and share the effects of each clone. Our second approach has been to develop methods that lower the cost of screens. We have done this by avoiding commercial kits and relying on component plastics, resins, and chemicals. We have found these methods to greatly lower the costs of doing screens, and in many cases, we have produced methods that are superior to equivalent commercial products. Finally, we have developed methods that utilize viral infections as a method to induce both cDNA and shRNA constructs. At present, our favored vectors are all retrovirus-based, but other viral vectors will likely be valuable as well. These approaches have led us to believe that essentially any characteristic of a cell can be explored using high-throughput function screens as long as a robust assay format can be designed.

\section{ACKNOWLEDGMENTS}

The authors are deeply indebted to W. Hahn, N. Hacohen, D. Root, D. Sabatini, E. Lander, and all members of the RNAi Consortium for providing a portion of their RNAi repository prior to publication; to Jeng Shin-Lee and R. Mulligan for advice on retroviral cloning and preparation and for access to the retroviral backbone pMMPf2. In addition, the authors thank Lance K. Ching for excellent technical assistance, as well as all of the members of the Harvard Institute of Proteomics for their comments and recommendations. This work was supported in part by a sponsored research agreement from Astra Zeneca.

\section{REFERENCES}

Bardelli A., Parsons D.W., Silliman N., Ptak J., Szabo S., Saha S., Markowitz S., Willson J.K., Parmigiani G., Kinzler K.W., Vogelstein B., and Velculescu V.E. 2003. Mutational analysis of the tyrosine kinome in colorectal cancers. Science 300: 949.

Brizuela L., Richardson A., Marsischky G., and LaBaer J. 2002. The FLEXGene repository: Exploiting the fruits of the genome projects by creating a needed resource to face the challenges of the post-genomic era. Arch. Med. Res. 33: 318.

Brummelkamp T.R., Bernards R., and Agami R. 2002. A system for stable expression of short interfering RNAs in mammalian cells. Science 296: 550.

Carpenter A.E. and Sabatini D.M. 2004. Systematic genomewide screens of gene function. Nat. Rev. Genet. 5: 11.

Carter P., Presta L., Gorman C.M., Ridgway J.B., Henner D., Wong W.L., Rowland A.M., Kotts C., Carver M.E., and Shepard H.M. 1992. Humanization of an anti-p185HER2 antibody for human cancer therapy. Proc. Natl. Acad. Sci. 89: 4285.

Cobleigh M.A., Vogel C.L., Tripathy D., Robert N.J., Scholl S., Fehrenbacher L., Wolter J.M., Paton V., Shak S., Lieberman G., and Slamon D.J. 1999. Multinational study of the efficacy and safety of humanized anti-HER 2 monoclonal antibody in women who have HER2-overexpressing metastatic breast cancer that has progressed after chemotherapy for metastatic disease. J. Clin. Oncol. 17: 2639.

Cunningham D., Humblet Y., Siena S., Khayat D., Bleiberg H., Santoro A., Bets D., Mueser M., Harstrick A., Verslype C., Chau I., and Van Cutsem E. 2004. Cetuximab monotherapy and cetuximab plus irinotecan in irinotecan-refractory metastatic colorectal cancer. N. Engl. J. Med. 351: 337.

Davies H., Bignell G.R., Cox C., Stephens P., Edkins S., Clegg 
S., Teague J., Woffendin H., Garnett M.J., Bottomley W., Davis N., Dicks E., Ewing R., Floyd Y., Gray K., Hall S., Hawes R., Hughes J., Kosmidou V., Menzies A., Mould C., Parker A., Stevens C., Watt S., and Hooper S., et al. 2002. Mutations of the BRAF gene in human cancer. Nature 417: 949.

Druker B.J., Talpaz M., Resta D.J., Peng B., Buchdunger E., Ford J.M., Lydon N.B., Kantarjian H., Capdeville R., OhnoJones S., and Sawyers C.L. 2001. Efficacy and safety of a specific inhibitor of the BCR-ABL tyrosine kinase in chronic myeloid leukemia. N. Engl. J. Med. 344: 1031.

Forsburg S.L. 2001. The art and design of genetic screens: Yeast. Nat. Rev. Genet. 2: 659.

Hafen E., Basler K., Edstroem J.E., and Rubin G.M. 1987. Sevenless, a cell-specific homeotic gene of Drosophila, encodes a putative transmembrane receptor with a tyrosine kinase domain. Science 236: 55.

Hannon G.J. 2002. RNA interference. Nature 418: 244.

Hannon G.J. and Rossi J.J. 2004. Unlocking the potential of the human genome with RNA interference. Nature 431: 371.

Hartwell L.H., Culotti J., and Reid B. 1970. Genetic control of the cell-division cycle in yeast. I. Detection of mutants. Proc. Natl. Acad. Sci. 66: 352.

Hartwell L.H., Culotti J., Pringle J.R., and Reid B.J. 1974. Genetic control of the cell division cycle in yeast. Science 183: 46.

Hunter T. 2000. Signaling-2000 and beyond. Cell 100: 113.

Inoue H., H. Nojima, and H. Okayama. 1990. High efficiency transformation of Escherichia coli with plasmids. Gene 96: 23.

Jacks T., Remington L., Williams B.O., Schmitt E.M., Halachmi S., Bronson R.T., and Weinberg R.A. 1994. Tumor spectrum analysis in p53-mutant mice. Curr. Biol. 4: 1.

Lynch T.J., Bell D.W., Sordella R., Gurubhagavatula S., Okimoto R.A., Brannigan B.W., Harris P.L., Haserlat S.M., Supko J.G., Haluska F.G., Louis D.N., Christiani D.C., Settleman J., and Haber D.A.. 2004. Activating mutations in the epidermal growth factor receptor underlying responsiveness of non-small-cell lung cancer to gefitinib. N. Engl. J. Med. 350: 2129.

Moffat J. and Sabatini D.M. 2006. Building mammalian signalling pathways with RNAi screens. Nat. Rev. Mol. Cell Biol. 7: 177.
Moffat J. Grueneberg D.A., Yang X., Kim S.Y., Kloepfer A.M., Hinkle G., Piqani B., Eisenhaure T.M., Luo B., Grenier J.K., Carpenter A.E., Foo S.Y., Stewart S.A., Stockwell B.R., Hacohen N., Hahn W.C. Lander E.S., Sabatini D.M., and Root D.E. 2006. A lentiviral RNAi library for human and mouse genes applied to an arrayed viral high-content screen. Cell 124: 1283.

Moon R.T., Kohn A.D., De Ferrari G.V., and Kaykas A.. 2004. WNT and beta-catenin signalling: Diseases and therapies. Nat. Rev. Genet. 5: 691.

Nurse P., Masui Y., and Hartwell L.. 1998. Understanding the cell cycle. Nat. Med. 4: 1103.

Paddison P.J., Caudy A.A., Sachidanandam R., and Hannon G.J. 2004. Short hairpin activated gene silencing in mammalian cells. Methods Mol. Biol. 265: 85.

Paez J.G., Janne P.A., Lee J.C., Tracy S., Greulich H., Gabriel S., Herman P., Kaye F.J., Lindeman N., Boggon T.J., Naoki K., Sasaki H., Fujii Y., Eck M.J., Sellers W.R., Johnson B.E., and Meyerson M.. 2004. EGFR mutations in lung cancer: Correlation with clinical response to gefitinib therapy. Science 304: 1497.

Park J., Hu Y., Murthy T.V., F. Vannberg, Shen B., Rolfs A., Hutti J.E., Cantley L.C., LaBaer J., E. Harlow, and L. Brizuela. 2005. Building a human kinase gene repository: Bioinformatics, molecular cloning, and functional validation. Proc. Natl. Acad. Sci. 102: 8114.

Pearlberg J. and LaBaer J. 2004. Protein expression clone repositories for functional proteomics. Curr. Opin. Chem. Biol. 8: 98.

Rual J.F., Hirozane-Kishikawa T., Hao T., Bertin N., Li S., Dri$\cot$ A., Li N., Rosenberg J., Lamesch P., Vidalain P.O., Clingingsmith T.R., Hartley J.L., Esposito D., Cheo D., Moore T., Simmons B., Sequerra R., Bosak S., Doucette-Stamm L., Le Peuch C., Vandenhaute J., Cusick M.E., Albala J.S., Hill D.E., and Vidal M. 2004. Human ORFeome version 1.1: A platform for reverse proteomics. Genome Res. 14: 2128.

Simon M.A., Bowtell D.D., Dodson G.S., Laverty T.R., and Rubin G.M. 1991. Ras1 and a putative guanine nucleotide exchange factor perform crucial steps in signaling by the sevenless protein tyrosine kinase. Cell 67: 701.

Xie K. and Abbruzzese J.L. 2003. Developmental biology informs cancer: The emerging role of the hedgehog signaling pathway in upper gastrointestinal cancers. Cancer Cell 4: 245. 


\section{$\$_{\text {SSH\& }}^{\infty} \mathrm{CS}$ Cold Spring Harbor Symposia SYMPOSIA on Quantitative Biology}

\section{Screens Using RNAi and cDNA Expression as Surrogates for Genetics in Mammalian Tissue Culture Cells}

J. PEARLBERG, S. DEGOT, W. ENDEGE, et al.

Cold Spring Harb Symp Quant Biol 2005 70: 449-459

Access the most recent version at doi:10.1101/sqb.2005.70.047

References This article cites 30 articles, 10 of which can be accessed free at: http://symposium.cshlp.org/content/70/449.full.html\#ref-list-1

License

Email Alerting Receive free email alerts when new articles cite this article - sign up in Service the box at the top right corner of the article or click here. 